\title{
Identification of novel nuclear localization signal within the ErbB-2 protein
}

\author{
Qiao Qiao CHEN, Xiao Ying CHEN, Yun Yun JIANG, Jing LIU* \\ School of Life Science, University of Science and Technology of China, Hefei 230027, China
}

\begin{abstract}
ErbB2, a member of the receptor tyrosine kinase family, is frequently over-expressed in breast cancer. Proteolysis of the extracellular domain of ErbB2 results in constitutive activation of ErbB2 kinase. Recent study reported that ErbB2 is found in the nucleus. Here, we showed that ErbB2 is imported into the nucleus through a nuclear localization signal (NLS)-mediated mechanism. The NLS sequence KRRQQKIRKYTMRR (aa655-668) contains three clusters of basic amino acids and it is sufficient to target GFP into the nucleus. However, mutation in any basic amino acid cluster of this NLS sequence significantly affects its nuclear localization. Furthermore, it was found that this NLS is essential for the nuclear localization of ErbB2 since the intracellular domain of Erb2 lacking NLS completely abrogates its nuclear translocation. Taken together, our study identified a novel nuclear localization signal and reveals a novel mechanism underlying ErbB2 nuclear trafficking and localization.
\end{abstract}

Keywords: ErbB2, NLS, intracellular domain.

\section{INTRODUCTION}

The ErbB family of receptor tyrosine kinases, including the EGF receptor (EGFR), ErbB2/HER2/Neu, ErbB3 and ErbB4, regulates cellular proliferation, differentiation, and programmed cell death in a growth factor-dependent manner [1]. The proto-oncogene ErbB2 encodes a 185KDs protein (P185) that is over-expressed in $25-30 \%$ of the patients with breast cancer and part of the patients with ovarian, lung, gastric, and oral cancers [2,3]. ErbB2, like other ErbB family numbers, contains an extracellular domain (ECD, amino acids 1-632), a hydrophobic transmembrane domain (TM, amino acids 633-654), and an intracellular domain (ICD, amino acids 655-1234) [4]. Ligand binding is thought to initiate the formation of homoand hetero-dimers with other ErbB members [5]. However, a distinguishing characteristic of ErbB2 is that it lacks a ligand. Instead, ErbB2 tends to form hetero-dimer with other ErbB family members to generate high-affinity complexes for different ligands [6]. This process is followed by intrinsic tyrosine kinase-mediated autophosphorylation and ultimately results in activated receptor complexes and downstream signaling cascade [7].

Although it is commonly known that tyrosine kinase

*Correspondence: Jing LIU

Tel: +86-551-3606294; Fax: +86-551-3601437;

E-mail: jliu@ustc.edu.cn receptors such as the ErbB family function as signaling initiators on the cell membrane, evidences have begun to accumulate that receptors or fragments of receptors travel from the plasma membrane to the nucleus by different mechanisms and may act as a kinase or transcription factor in the nucleus [8-10]. The reported tyrosine kinase receptors include four members of ErbB family [11-15] and other tyrosine kinase receptors like VEGF receptor [16], FGF receptor $[17,18]$ and NGF receptor [19].

However, the four members of ErbB family were translocated in the nucleus in different form. In the case of EGFR, the complete ligand-full length receptor complex was reported to be present in the nucleus [11]. Nuclear ErbB3 was localized to the nucleus as full-length protein [13], while only the intracellular domain ErbB4 can be targeted to the nucleus $[14,15]$. In the case of ErbB2, it has been recently demonstrated that full-length ErbB2 is located to the nucleus by a combination of electron microscopy, fluorescence microscopy, immunohistochemistry and biochemical fraction [12]. However, a great deal of studies have shown that the extracellular domain (ECD) ErbB2 can be proteolyzed from the cell membrane, which results in constitutive activation of the remaining membrane-associated ErbB2 domain [20,21]. Monoclonal antibodies targeting the ErbB2 ectodomain, such as trastuzumab and 4D5, have been shown to abrogate the ErbB2 activation processes and to interfere with ErbB2-dependent gene expression. One of the mechanisms mediating these ef- 
fects is the prevention of ErbB2 ectodomain cleavage [22, 23]. Despite full-length ErbB2 was found in the nucleus, it was unclear whether the ICD of ErbB2 can also target to the nucleus like ErbB4 [12].

The subcellular location of an individual protein depends largely on whether its amino acid sequence contains sorting signals directing the protein to specific cellular location. Nuclear Localization Signals (NLS), which can direct protein through the nuclear pore complex, are characteristically rich in the basic amino acids lysine and arginine [24]. In ErbB family, nuclear localization signals have been identified within ErbB3 [13], ErbB4 [1], and a putative juxtamembrane EGFR NLS was shown to mediate nuclear translocation of $\beta$-galactosidase[11]. Although the nuclear translocation of ErbB2 has been reported [12, 25], the molecular mechanism underlying the nuclear targeting of ErbB2 remains elusive.

Here we demonstrated that a novel nuclear localization signal exists within ErbB2 ( ${ }^{655}$ KRRQQKIRKYTMRR $\left.{ }^{668}\right)$. Fusing this NLS to GFP drove the resulting fusion protein into the nucleus. Mutation of any basic amino acid residues in this region minimizes the nuclear localization of ErbB2.

\section{MATERIALS AND METHODS \\ ErbB-2 cDNA}

The human ErbB-2 cDNA used in these experiments has been cloned into the vector $\mathrm{pBaBe}$ that was a gift from Dr. Greene MI (School of Medicine, University of Pennsylvania, Philadelphia)

\section{Oligonucleotides}

All primers used in this study are listed in Tab. 1. Restriction enzyme sites are underlined.

\section{Plasmid construction}

Using plasmid $\mathrm{pBaBe}$ bearing the ErbB-2 gene as a template, primer pairs Pnb1/Pcs580, Pnb1/Pcs45p, Pnb1/Pcs35, Pnb1/Pcs25, Pnb1/Pcs100, Pnb15/Pcs45, Pnb15/Pcs580 and Pnb15/Pcs100 were used for amplification of the corresponding ErbB2 fragments and inserted into the SalI/BglII digested vector pEGFP-C1 ( BD Bioscience Clontech, Palo Alto, CA). Then eight recombinant plasmids are generated as follows: GFP-ErbB2 $\Delta 655-1234$, GFP-ErbB2 $\Delta 655$ 699, GFP-ErbB2 $\Delta 655-689$, GFP-ErbB2 $\Delta 655-679$, GFP-ErbB2 $\Delta 655$ 754, GFP-ErbB2 $\Delta 669-699$, GFP-ErbB2 $\Delta 669-1234$ and GFPErbB2 $\Delta 669-754$.

Since the gene fragment of 14 amino acid sequence NLS is short, we chose a MluI site out of MCS of pEGFP-C1 vector. The 14amino acid sequence containing the putative NLS of ErbB2 was fused to GFP using an MluI site as 3' enzyme site. To construct this plasmid GFP-ErbB2 $\Delta 655-668$, the pEGFP-C1 was firstly amplified in a PCR using forward Pn14-1 and reverse Pcm14. The PCR product was then used as a template with forward primer Pnb14-2 and reverse primer Pcm14 [26]. The final PCR product was digested with BglII/MluI and ligated to pEGFP-C1 vector digested with BgIII/ MluI.

The constructs Mut1 and Mut2 are generated by ligation of the
Tab. 1 All the primers used in this study are list as follows

\begin{tabular}{|c|c|}
\hline $\begin{array}{l}\text { Primer } \\
\text { name }\end{array}$ & Sequence \\
\hline Pnb1 & 5'-CCGAGATCTAAGCAGCGGCAGCAG-3' \\
\hline Pnb15 & 5'-CCGAGATCTCTGCTGCAGGAAACGGAG-3' \\
\hline Pn14-1 & $\begin{array}{l}\text { 5'-GATCCGGAAGTACACGATGCGGAGATAAC } \\
\text { GAGCTCAAGCTTCGAATTCT-3' }\end{array}$ \\
\hline Pnb14-2 & $\begin{array}{l}\text { 5'-CCGAGATCTAAGCGACGGCAGCAGAAGAT } \\
\text { CCGGAAGTACACGATG-3' }\end{array}$ \\
\hline Pcm14 & 5'-CAATTTACGCGTTCAGATACATTGATG-3' \\
\hline $\operatorname{Pcs} 25$ & $\begin{array}{l}\text { 5'-CCGACTGTCGACTCACAGCGGCTCCACCA } \\
\text { GCTC-3' }\end{array}$ \\
\hline $\operatorname{Pcs} 35$ & 5'-CCGGTCGACTCACGCCTGGTTGGGCATCGC-3' \\
\hline $\operatorname{Pcs} 45$ & 5'-CCGGTCGACTCACAGCTCCGTCTCTTTCAG-3' \\
\hline$P \operatorname{cs} 100$ & 5'-CCGGTCGACTCAAGCCATCACGTATGCTTC-3' \\
\hline$P \operatorname{cs} 580$ & 5'-CCGGTCGACTCACACTGGCACGTCCAG-3' \\
\hline Pmutlb & $\begin{array}{l}\text { 5'-CCGAGATCTACGGGAGGGCAGCAGAAGAT } \\
\text { CCGGAAG-3' }\end{array}$ \\
\hline Pmut2b & $\begin{array}{l}\text { 5'-CCGAGATCTAAGCGACGGCAGCAGACGAT } \\
\text { CGGGACGTACACGATG-3' }\end{array}$ \\
\hline Pmut3-1 & $\begin{array}{l}\text { 5'-ATCCGGAAGTACACGATGGGGGGACTGCT } \\
\text { GCAGGAAACG-3' }\end{array}$ \\
\hline Pmut3-2b & 5'-CCGAGATCTAAGCGACGGCAGCAGAAATC \\
\hline
\end{tabular}

PFlagDn 5'-CCGAGATCTGAAGCGACGGCAGCAGAAG-3' PFlagDdn 5'-CCGAGATCTGCTGCTGCAGGAAACGGAG-3' Restriction enzyme sites are underlined.

BglII/SalI digested PCR product from amplified $\mathrm{pBaBe}$ using primer pairs Pmut1b/Pcs45, Pmut2b/Pcs45, respectively, and the corresponding BglII/SalI digested pEGFP vector.

To construct plasmid Mut3, we first amplified $\mathrm{pBaBe}$ with primer pair Pmut3-1/Pcs45 while this PCR product was used as template and amplified with primer pair Pmut3-2b/Pcs45. The PCR product was digested with BglII/SalI and ligated to the corresponding pEGFP vector.

The constructs Flag-ICD and Flag-ICD $\Delta$ NLS are generated by ligation of the BglII/SalI digested PCR product from amplified $\mathrm{pBaBe}$ using primer pairs PFlagDn /Pcs45, PFlagDdn/Pcs45, respectively, and the corresponding BglII/SalI digested p3XFLAG-myc-CMV ${ }^{\mathrm{TM}}$ 24 vector (Sigma).

The correct orientation and insertion of fragments in each of the constructs were confirmed by DNA sequencing.

\section{Cell lines and transfections}

Monkey kidney fibroblasts COS-7cells (Type Culture Collection of Chinese Academy of Science) were maintained in Dulbecco's modified Eagle's medium supplemented with $10 \%$ calf BS, $100 \mu \mathrm{g} / \mathrm{ml}$ penicillin and 100 units/ml streptomycin. Human cervix adenocarcinoma HeLa cells (Type Culture Collection of Chinese Academy of 
Science) were grown in Eagle's minimum essential medium supplemented with $10 \%$ calf BS, $100 \mu \mathrm{g} / \mathrm{ml}$ penicillin, 100 units $/ \mathrm{ml}$ streptomycin. Transfection was carried out using Lipofectamine (Invitrogen) according to the manufacturer's specifications.

\section{Visualization of GFP fluorescence}

Cells were observed on dishes with an inversed fluorescence microscope (Olympus IX70). Fluorescence images were captured using Viewfinder Lite 1.0 (magnification $\times 200$ ).

\section{Immunofluorescence microscopy}

Cells were grown on acid-treated glass coverslips. After transfection, cells were washed twice with PBS. Permeabilization was carried out for $3 \mathrm{~min}$ in $0.1 \%$ Triton X-100 at room temperature. Pre-extracted cells were fixed in $3.7 \%$ paraformaldehyde for $10 \mathrm{~min}$. After washing three times with PBS, cells were blocked with $1 \%$ BSA (Sigma) in PBS containing $0.05 \%$ Tween-20 for $1 \mathrm{~h}$, and then incubated with primary antibodies for $1 \mathrm{~h}$ followed by secondary antibodies for $1 \mathrm{~h}$. Slides were examined with a Zeiss Axiovert-200 fluorescence microscope, and images were collected and analyzed with Image-5 (Carl Zeiss, Germany).

\section{RESULTS}

ErbB2 intracellular domain is located in the nucleus

Studies on the ErbB2 extracellular domain (ECD) shedding revealed that the activation of remaining membraneassociated domain led to increased signal transduction [20], suggesting that the intracellular domain of ErbB2 might target to the nucleus like that of ErbB4.

To test our hypothesis, fusion protein comprising green fluorescent protein (GFP) at NH2-terminus of the ErbB2 intracellular domain (Fig. 1A) were transiently expressed in HeLa cells. Interestingly, the fusion protein (molecular weight $90 \mathrm{kDa}$; GFP-ICD) was indeed localized to the nucleus (Fig. 1B; c, d). To validate the specificity of this phenotype, we carried out our experiment using a different cell line. As shown in Fig. 1C, GFP-ICD is also translocated into the nucleus in COS-7 cells. Given the fact that nuclear pore limits the passive translocation of any passenger protein greater than $60 \mathrm{kDa}$, the uptake of the GFP-ICD fusion protein $(90 \mathrm{kDa})$ is most likely via NLS located within the ICD of ErbB2.

\section{Identification of a Nuclear Localization Signal in ErbB2}

Since nuclear localization is usually mediated by NLS within the proteins, we therefore analyzed the ErbB2 protein sequences for confirmation of the presence of NLS using softwares PSORT II (http://psort.nibb.ac.jp) and PredictNLS (http://cubic.bioc.columbia.edu/predictNLS). Although analyses using PSORTII and PredictNLS did not reveal any typical NLS, we found a sequence KRRQQKIR KYTMRR (amino acids 655 to 668) in juxtamembrane intracellular domain of ErbB2 that is similar to the NLS of

\section{A GFP-ErbB2 $4655-1234$

$\begin{array}{rl}\text { GFP NLS } & \\ 655669 & 1234\end{array}$
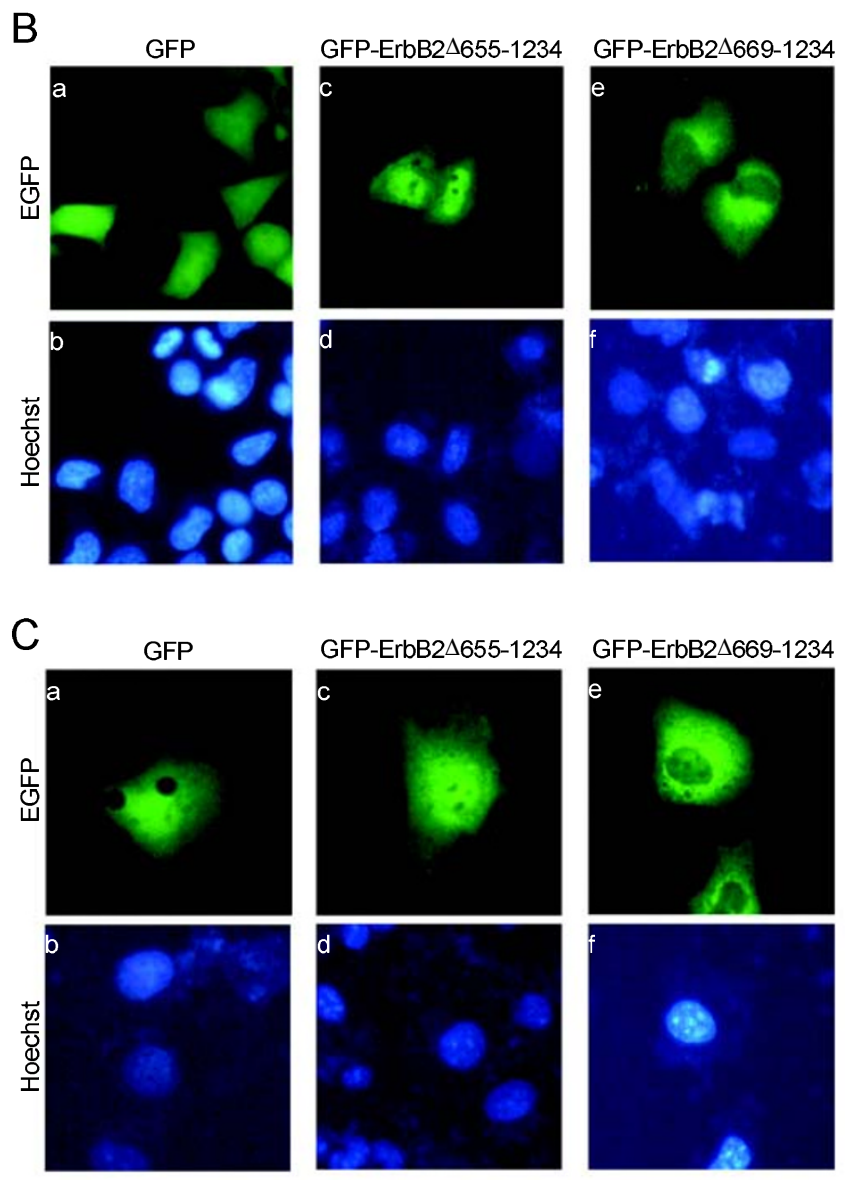

Fig. 1 The intracellular domain of ErbB2 is targeted and localized to the nucleus. (A) Schematic presentation of ErbB2 intracellular domain fused to GFP. NLS within ErbB2 is indicated in black block. (B) HeLa were transiently transfected with GFP (a, b), GFPErbB2 $\Delta 655-1234(\mathrm{c}, \mathrm{d})$ and GFP-ErbB2 $\Delta 669-1234$ (e, f). The pictures were taken $20 \mathrm{~h}$ after transfection in flurescent field. Nuclei were stained with Hoechst dye. (C) COS-7 cells were transiently transfected with GFP $(\mathrm{a}, \mathrm{b}), \mathrm{GFP}-\mathrm{ErbB} 2 \Delta 655-1234(\mathrm{c}, \mathrm{d})$ and GFPErbB2 $\Delta 669-1234$ (e, f).

ErbB4 (RRKSIKKKRALRR; amino acids 676-688) and putative NLS of EGFR (RRRHIVRKRTLRR; amino acids 669-681), which are also located to the same cytoplasmic juxtamembrane domain of each protein respectively. The sequence analysis led us to hypothesize the aforementioned sequence is a putative NLS of ErbB2.

To validate our hypothesis on the ErbB2 NLS, we constructed a GFP-ErbB2 plasmid containing the putative NLS 
A

GPP

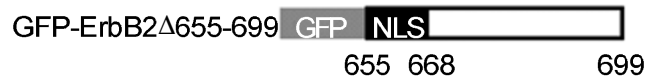

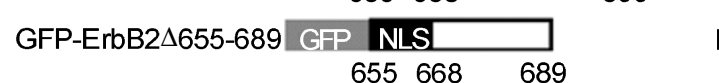

GFP-ErbB2 $\Delta 655-679$ GFP NLS

655668679

GFP-ErbB2 $\Delta 655-668$ GFP $\underset{65}{\text { NLS }}$

GFP-ErbB2 $\Delta 669-699$ GFP

669

C

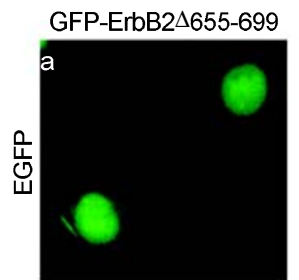

GFP-ErbB2 $\triangle 669-699$
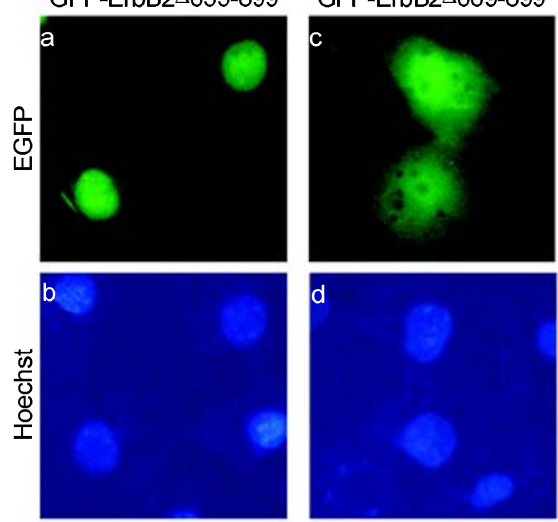

Subcellular localization

$\mathrm{N}+\mathrm{C}$

N

N

N

$\mathbf{N}$

$\mathrm{N}+\mathrm{C}$

699
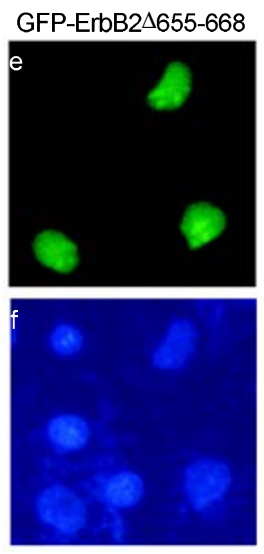

sequence (GFP-ErbB2 $\triangle 655-699)$ and transfected it into HeLa and COS-7 cells, respectively. To map a minimal sequence in ErbB2 which is responsible for ErbB2 targeting to the nucleus, we constructed several GFP-tagged ErbB2 fragments (Fig. 2A) and expressed those constructs in HeLa cells. Examination of GFP fusion protein localization reveals that the putative NLS containing 14 amino acids is sufficient to traffic to the nucleus (Fig. 2B, e, f; Fig. $2 \mathrm{C}, \mathrm{e}, \mathrm{f}$ ). To determine if this putative NLS is essential for targeting ErbB2 to the nucleus, we deleted the NLS (655-668) and generated construct GFP-ErbB2 $\Delta 669-699$. As shown in Fig. 2B (c, d) and Fig. 2C (c, d), elimination of the putative NLS resulted in diffused distribution of GFP-ErbB2 in both cytoplasm and the nucleus. Similarly, expression of ErbB2 ICD without NLS (GFP-ErbB2 $\Delta 669$ 1234) accumulates in the cytoplasm only (Fig. 1B, e, f; $1 \mathrm{C}, \mathrm{e}, \mathrm{f})$, indicating that the putative NLS is required for the nuclear localization of ErbB2. To validate the specificity of this phenotype, we carried out our experiment using

B
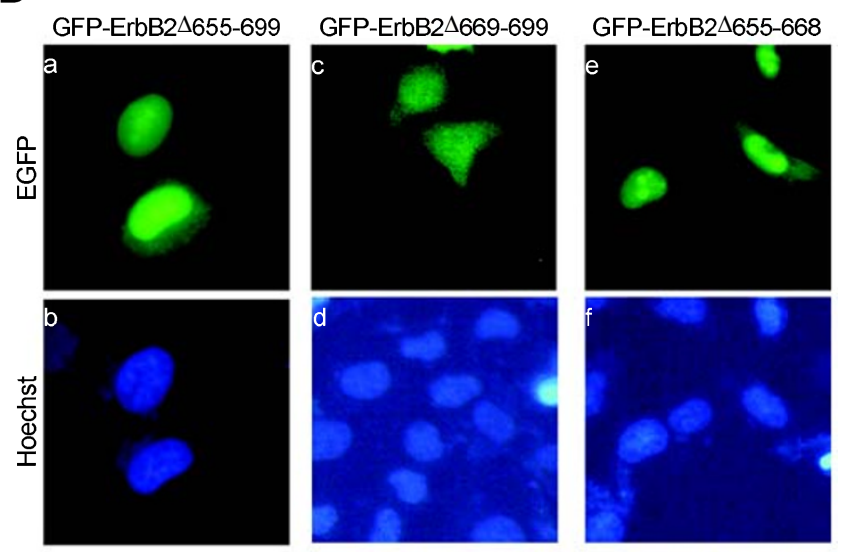

Fig. 2 Identification of a novel nuclear localization signal peptide in ErbB2. (A) Schematic representation of a series of ErbB2 fragments fused to GFP and their subcellular localization (N: Nuclear; $\mathrm{C}$ : Cytoplasm). (B) HeLa cells were transiently transfected with GFPErbB2 $\Delta 655-699$ (a, b), GFP-ErbB2 $\Delta 669-699(\mathrm{c}, \mathrm{d})$ and GFPErbB2 $\Delta 655-668$ (e, f). (C) COS-7 cells were transiently transfected with GFP-ErbB2 $\Delta 655-699$ (a, b), GFP-ErbB2 $\Delta 669-699(\mathrm{c}, \mathrm{d})$ and GFP-ErbB2 $\Delta 655-668$ (e, f).

a different tag (Fig. 3A). As shown in Fig. 3B, FLAG-ICD is also translocated into the nucleus and FLAG-ICD $\triangle$ NLS accumulates in the cytoplasm only. Thus, we conclude that the 14 aa NLS (KRRQQKIRKYTMRR) is essential for the nuclear localization of ErbB2 ICD.

\section{Role of basic amino acid residues in nuclear localiza- tion of ErbB2}

It is generally believed that NLS can be categorized into at least three different types. The first type of NLS is a continuous stretch of amino acids, which also called monopartite. The second type of NLS is a bipartite sequence, which contains two clusters of basic amino acid residues separated by an intervening 10-12 aa spacer. The third type is non-classical NLS that do not contain well-conserved sequence [24]. The NLS sequence of ErbB2 KRRQQKIRKYTMRR contains three clusters of two or three continuous basic amino acid residues. To determine precisely which cluster is responsible for the nuclear localization, site-directed mutants in the basic residues within each basic amino acid cluster were generated (Fig. 4A). A list of the three resultant NLS mutations was shown in Tab. 2. All the three mutants were based on the construct of GFP-ErbB2 $\Delta 655-699$. As shown in Fig. 4B, substitution of basic amino acids within the NLS disrupted the nuclear localization of ErbB2 in HeLa and effected a 
Tab. 2 Summary of ErbB2 NLS mutants

\begin{tabular}{|c|c|}
\hline NLS & Sequence \\
\hline WT & 655 KRRQQKIIRKYTMRR 668 \\
\hline Mut1 & $\overline{\text { TGGQQK}} \underline{\underline{R K}} Y$ TM $\underline{\overline{R R}}$ \\
\hline Mut2 & KRRQQTIGTYTMR $\overline{\text { RR }}$ \\
\hline Mut3 & KRRQQKIRKYTMGG \\
\hline
\end{tabular}

Amino acid residues are indicated with the single-letter code. Bold letters indicate the mutated amino acid residues within NLS. The basic amino acids are underlined.
A

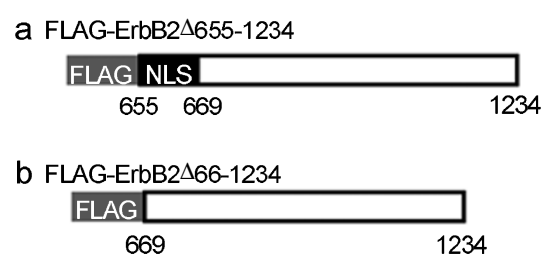

B
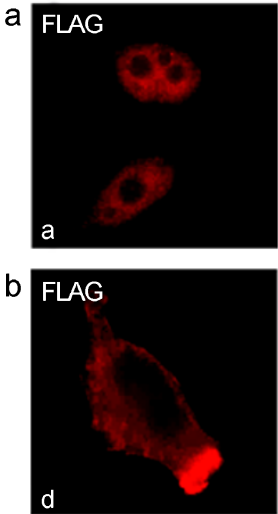

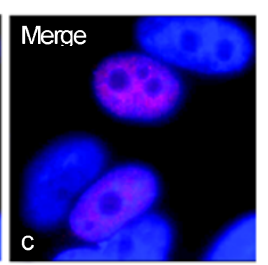

Merge

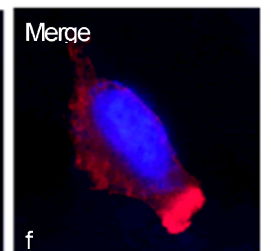

Fig. 3 The NLS is essential for the nuclear localization of ErbB2 ICD. (A) Schematic presentation of ErbB2 intracellular domain and ErbB2 ICD $\triangle$ NLS fused to FLAG. (B) HeLa were transiently transfected with FLAG-ErbB2ICD and FLAG-ErbB2ICD $\triangle$ NLS. At $24 \mathrm{~h}$ after transfection, cell were extracted, fixed and processed for immunocytochemistry. (a-c) Images collected from one Hela cell double stained for anti-FLAG antibody (ErbB2 ICD, red), DAPI (DNA, blue) and their merged images. (d-f) Images collected from one Hela cell double stained for anti-FLAG antibody (ErbB2 ICD $\triangle$ NLS, red), DAPI (DNA, blue) and their merged images.

Tab. 3 Comparison of identified NLS in the ErbB family

\begin{tabular}{cll}
\hline Protein name & \multicolumn{2}{c}{ Identified NLS } \\
\hline ErbB1 & 645 & RRRHIVRKRTLRR 657 \\
ErbB2 & 655 & KRRQQKIRKYTMRR 668 \\
ErbB3 & 1202 & RRRRHSP 1208 \\
ErbB4 & 676 & RRKSIKKKRALRR 688 \\
\hline
\end{tabular}

Amino acid residues are indicated with the single-letter code. Bold letters indicate the basic amino acids. The NLS reported in this study is underlined.
A

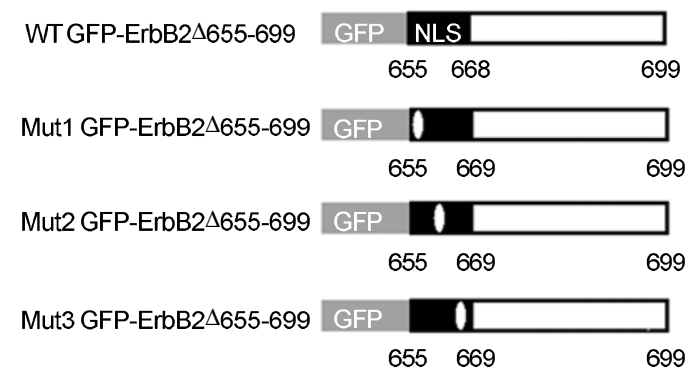

B
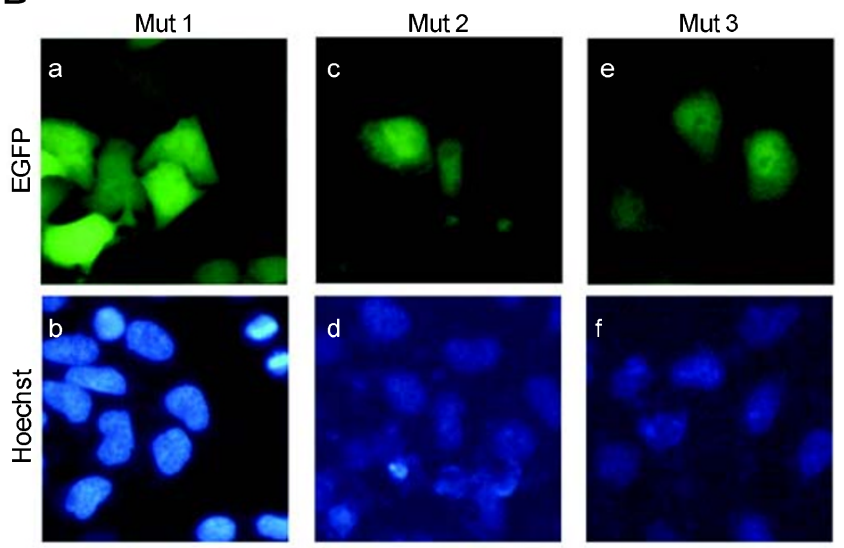

Fig. 4 Subcellular localization of GFP-ErbB2 $\Delta 655-699$ following mutation of NLS sequence. (A) Schematic presentation of three NLS mutants of GFP-ErbB2 $\Delta 655-699$. The mutation sites are indicated in white spot. (B) Hela cells were transiently transfected with constructs of Mut1 (a, b), Mut2 (c, d), Mut3 (e, f).

diffused cytoplasmic distribution, indicating that all three basic amino acid clusters are essential for the nuclear localization of ErbB2. Thus, we conclude that the NLS of ErbB2 seems belongs to a non-classical NLS, neither bipartite nor monopartite.

\section{DISCUSSION}

Although nuclear localization of the four ErbB family members has been described recently, the functional NLS was reported in EGFR, ErbB3 and ErbB4 but not in Erb2. Our identification of NLS in ErbB2 indicated that nuclear translocation by NLS is a common phenomenon for all ErbB family members perhaps includes the whole tyrosine kinase receptor family. We summarize identified amino acid sequences of all four NLS in ErbB family in Tab. 3. The functional significance of cell surface receptor nuclear translocation was exemplified by the recent studies including the interaction of nuclear EGFR with cyclin D1 promoter [11], the association of nuclear ErbB2 with COX-2 promoter [12], and the regulation of STAT5A by ErbB4 
[1]. However, the four members of ErbB family appear to use different mechanisms for their nuclear translocation. In the case of ErbB4, the intracellular domain was reported to be present in the nucleus $[14,15]$. It was recently reported that full-length ErbB2, like EGFR and ErbB3, translocates to the nucleus [12]. However, our expression of full-length ErbB2 in COS-7 cells revealed no obvious nuclear localization but instead a typical plasma membrane surface distribution. Large collection of experimental evidence demonstrated that ErbB2 undergoes proteolytical cleavage of its extracellular domain (ECD) which yields an activated intracellular domain. Since the ECD proteolysis of ErbB2 is similar to that of ErbB4, we therefore proposed that ErbB2 ICD may translocate into the nucleus like ErbB4 and exerts its nuclear function. Our identification of functional NLS in ErbB2 suggests that nuclear translocation is a general phenomenon underlying ErbB family members, which may also be relevant for EGFR nuclear translocation. The ErbB2 NLS, the putative EGFR NLS and ErbB4 NLS share basic residue stretches positioned immediately downstream of the transmembrane domain. Unlike the other ErbB family members, ErbB3 does not have a functional tyrosine kinase domain. So, the position of ErbB3 NLS is different from the other members of the ErbB family. These studies suggest that the nuclear activities of ErbB family, except for ErbB3, are functionally and/or mechanistically similar.

We demonstrated above that the $\mathrm{N}$ terminus of ErbB2 intracellular domain harbors an unconventional NLS $\left({ }^{655}\right.$ KRRQQKJRKYTMRR $\left.{ }^{668}\right)$ that controls its nuclear localization. Mutation analysis revealed that the basic amino acid clusters in this NLS is required for the nuclear localization of GFP-ErbB2 fusion proteins. The requirement of all three basic amino acid clusters for the nuclear localization of ErbB2 establishes a novel class of NLS. Given the importance of ErbB2 nuclear signaling cascade in the development and progression of breast cancer, it would be of great interests to explore whether modulation of this 14 amino acid NLS by small molecules could intercept the nuclear localization of ErbB2 in cancer cells.

Taken together, our study reveals a novel class of NLS in ErbB2 which is essential for targeting intracellular domain of ErbB2 to the nucleus. The identification of NLS in ErbB2 provides molecular basis account for the mechanism underlying ErbB2 nuclear trafficking and function.

\section{ACKNOWLEDGEMENTS}

We thank Prof. Xue Biao YAO for critical review of this manuscript and Dr. Yong Hui YANG for his helpful suggestion and guidance during this study. This work was supported by Hi-Tech Research and Development Program of China (2004AA215260).
Received, Mar 28, 2005

Revised, May 25, 2005

Accepted, June 1, 2005

\section{REFERENCES}

1 Williams CC, Allison JG, Vidal GA, et al. The ERBB4/HER4 receptor tyrosine kinase regulates gene expression by functioning as a STAT5A nuclear chaperone. J Cell Biol 2004; 167:46978.

2 Hung MC, Matin A, Zhang Y, et al. HER-2/neu-targeting gene therapy-a review. Gene 1995; 159:65-71.

3 Slamon DJ, Clark GM, Wong SG, et al. Human breast cancer: correlation of relapse and survival with amplification of the HER2/neu oncogene. Science 1987; 235:177-82.

4 Ullrich A, Schlessinger J. Signal transduction by receptors with tyrosine kinase activity. Cell 1990; 61:203-12.

5 Tzahar E, Waterman H, Chen X, et al. A hierarchical network of interreceptor interactions determines signal transduction by Neu differentiation factor/neuregulin and epidermal growth factor. Mol Cell Biol 1996; 16:5276-87.

6 Graus-Porta D, Beerli RR, Daly JM, Hynes NE. ErbB-2, the preferred heterodimerization partner of all ErbB receptors, is a mediator of lateral signaling. EMBO J 1997; 16:1647-55.

7 Segatto O, Lonardo F, Pierce JH, Bottaro DP, Di Fiore PP. The role of autophosphorylation in modulation of erbB-2 transforming function. New Biol 1990; 2:187-95.

8 Carpenter G. Nuclear localization and possible functions of receptor tyrosine kinases- a review. Curr Opin Cell Biol 2003; 15: 143-8.

9 Wells A, Marti U. Signalling shortcuts: cell-surface receptors in the nucleus?-a review. Nat Rev Mol Cell Biol 2002; 3:697-702

10 Clevenger CV. Nuclear localization and function of polypeptide ligands and their receptors: a new paradigm for hormaone specificity within the mammary gland? Breast Cancer Res 2003; 5: 181-7.

11 Lin SY, Makino K, Xia W, et al. Nuclear localization of EGF receptor and its potential new role as a transcription factor. Nat Cell Biol 2001; 3:802-8.

12 Wang SC. Lien HC, Xia W, et al. Binding at and transactivation of the COX-2 promoter by nuclear tyrosine kinase receptor ErbB2. Cancer Cell 2004; 6:251-61.

13 Offerdinger M, Schofer C, Weipoltshammer K, Grunt TW. CerbB-3: a nuclear protein in mammary epithelial cells. J Cell Biol 2002; 157: 929-39.

14 Ni CY, Murphy MP, Golde TE, Carpenter G. $\gamma$-Secretase cleavage and nuclear localization of ErbB-4 receptor tyrosine kinase. Science 2001; 294: 2179-81.

15 Lee HJ, Jung KM, Huang YZ, et al. Presenilin-dependent ãsecretase-like intramembrane cleavage of ErbB4. J Biol Chem 2002; 277:6318-23.

16 Feng Y, Venema VJ, Venema RC, Tsai N, Caldwell RB. VEGF induces nuclear translocation of Flk-1/KDR, endothelial nitric oxide synthase, and caveolin-1 in vascular endothelial cells. Biochem Biophys Res Commun 1999; 256:192-7.

17 Reilly JF, Maher PA. Importin ß-mediated nuclear import of fibroblast growth factor receptor: role in cell proliferation. J Cell Biol 2001; 152:1307-12.

18 Maher PA. Nuclear translocation of fibroblast growth factor (FGF) receptors in response to FGF-2. J Cell Biol 1996; 134: 
529-36.

19 Rakowicz-Szulczynska EM, Herlyn M, Koprowski H. Nerve growth factor receptors in chromatin of melanoma cells, proliferating melanotytes, and colorectal carcinoma cells in vitro. Cancer Res 1988; 48:7200-6.

20 Baselga.J, Albanell.J. Mechanism of action of anti-HER2 monoclonal antibodies-a review. Ann Oncol 2001; 12: S35-41.

21 Condony-Servat J, Albanell J, Carlos lopez-Talavera J, Arribas J, Baselga J. Cleavage of the HER2 ectodomain is a pervanadateactivable process that is inhibited by the tissue inhibitor of metalloproteases-1 in breast cancer cells. Cancer Res 1999; 59: 1196-201.

22 Baselga J, Albanell J, Molina MA, Arribas J. Mechanism of action of trastuzumab and scientific update. Semin Oncol 2001; 28:4-11.

23 Yip YL, Ward RL. Anti-ErbB-2 monoclonal antibodies and ErbB2-directed vaccine. Cancer Immunol Immunother 2002; 50:56987.

24 Dingwall C, Laskey RA. Nuclear targeting sequences-a consensus? Trends Biochem Sci 1991; 16:478-81.
25 Xie Y, Hung MC. Nuclear localization of p185 neu tyrosine kinase and its association with transcriptional transactivation. Biochem Biophys Res Commun 1994; 203:1589-98.

26 Cheng LS, Liu AP, Yang JH, et al. Construction, expression and characterization of the engineered antibody against tumor surface antigen, p185 ${ }^{\mathrm{c}-\mathrm{erbB}-2}$. Cell Res 2003; 13:35-48.

27 Catez F, Erand M, Schaerer-Uthurralt N, et al. Unique motif for nucleolar retention and nuclear export regulated by phosphorylation. Mol Cell Biol 2002; 22:1126-39.

28 Rowland RR, Yoo D. Nucleolar-cytoplasmic shuttling of PRRSV nucleocapsid protein: a simple case of molecular mimicry or the complex regulation by nuclear import, nucleolar localization and nuclear export signal sequences-a review. Virus Res 2003; 95: 23-33.

29 Shun-Nu C, Lin CH, Lin A. An acidic amino acid cluster regulates the nucleolar localization and ribosome assembly of human ribosomal protein L22.FEBS Lett 2000; 484: 22-8.

30 Timmers AC, Stuger R, Schaap PJ, Van't Riet J, Raue HA. Nuclear and nucleolar localization of Saccharomyces cerevisiae ribosomal proteins S22 and S25. FEBS Lett 1999; 452: 335-40. 